\title{
Some new Fejér type inequalities via quantum calculus on finite intervals
}

\author{
Wengui Yang \\ School of Mathematics, Southeast University, Nanjing 210096, China \\ Ministry of Public Education, Sanmenxia Polytechnic, Sanmenxia 472000, China \\ e-mail: wgyang0617@yahoo.com
}

Received 2 Dec 2016

Accepted 7 May 2017

ABSTRACT: Some new inequalities of Fejér type for twice differentiable mappings are established via quantum calculus on finite intervals. The results presented are extensions of those given earlier.

KEYWORDS: integral inequalities, differentiable mappings, Chebyshev quantum integral inequality

MSC2010: 26D10 34A08 26D15

\section{INTRODUCTION}

The function $f:[a, b] \rightarrow \mathbb{R}$, is said to be convex if $f_{\alpha}(x, y) \leqslant \alpha f(x)+(1-\alpha) f(y)$ for all $x, y \in[a, b]$, $\alpha \in[0,1]$, where throughout the paper, $F_{q}(x, y)$ denotes $F(q x+(1-q) y)$. We say that $f$ is concave if $-f$ is convex.

Let $f: I \rightarrow \mathbb{R}$ be a convex mapping and $a, b \in I$ with $a<b$. The double inequality

$$
f\left(\frac{a+b}{2}\right) \leqslant \frac{1}{b-a} \int_{a}^{b} f(x) \mathrm{d} x \leqslant \frac{f(a)+f(b)}{2}
$$

is well known as Hermite-Hadamard's inequality for convex mapping ${ }^{1,2}$. Both inequalities hold in the reversed direction if $f$ is concave. We note that (1) may be regarded as a refinement of the concept of convexity and it follows easily from Jensen's inequality. Hermite-Hadamard's inequality has received renewed attention in recent years due the fact that it has been considered the most useful inequality for convex functions in mathematical analysis ${ }^{3-6}$. For example, Xi and $\mathrm{Qi}^{7}$ and Chun and $\mathrm{Qi}^{8}$ obtained some Hermite-Hadamard's inequalities for extended $s$-convex functions and functions whose third derivatives are convex, respectively. In Ref. 9 some Hermite-Hadamard type inequalities for $\left(p_{1}, h_{1}\right)-\left(p_{2}, h_{2}\right)$-convex functions on the coordinates were established. We now state some known results.

Theorem 1 (Ref. 10) Let $f: I \rightarrow \mathbb{R}$ be a convex mapping and $a, b \in I$ with $a<b$. Then

$$
\begin{aligned}
f\left(\frac{a+b}{2}\right) \int_{a}^{b} w(x) \mathrm{d} x & \leqslant \int_{a}^{b} f(x) w(x) \mathrm{d} x \\
& \leqslant \frac{f(a)+f(b)}{2} \int_{a}^{b} w(x) \mathrm{d} x
\end{aligned}
$$

where $w:[a, b] \rightarrow \mathbb{R}$ is nonnegative, integrable, and symmetric about $x=(a+b) / 2$, i.e., $w(x)=w(a+$ $b-x)$.

Theorem 2 (Refs. 11,12) Let $f:[a, b] \rightarrow \mathbb{R}$ be $a$ twice differentiable mapping such that there exist real constants $m$ and $M$ so that $m \leqslant f^{\prime \prime} \leqslant M$. Then

$$
\begin{aligned}
m \frac{(b-a)^{2}}{12} & \leqslant \frac{f(a)+f(b)}{2}-\frac{1}{b-a} \int_{a}^{b} f(x) \mathrm{d} x \\
& \leqslant M \frac{(b-a)^{2}}{12}
\end{aligned}
$$

and

$$
\begin{aligned}
m \frac{(b-a)^{2}}{24} & \leqslant \frac{1}{b-a} \int_{a}^{b} f(x) \mathrm{d} x-f\left(\frac{a+b}{2}\right) \\
& \leqslant M \frac{(b-a)^{2}}{24} .
\end{aligned}
$$

Theorem 3 (Ref. 13) Let $f:[a, b] \rightarrow \mathbb{R}$ be a twice differentiable mapping such that there exist real constants $m$ and $M$ so that $m \leqslant f^{\prime \prime} \leqslant M$. Then, for 
$\lambda \in[0,1]$,

$$
\begin{aligned}
& m \frac{\lambda(1-\lambda)}{2}(b-a)^{2} \\
& \quad \leqslant \lambda f(a)+(1-\lambda) f(b)-f_{\lambda}(a, b) \\
& \quad \leqslant M \frac{\lambda(1-\lambda)}{2}(b-a)^{2},
\end{aligned}
$$

and

$$
\begin{aligned}
& m \frac{(1-2 \lambda)^{2}}{8}(b-a)^{2} \\
& \quad \leqslant \frac{f_{\lambda}(a, b)+f_{\lambda}(b, a)}{2}-f\left(\frac{a+b}{2}\right) \\
& \quad \leqslant M \frac{(1-2 \lambda)^{2}}{8}(b-a)^{2} .
\end{aligned}
$$

Theorem 4 (Ref. 13) Let $f:[a, b] \rightarrow \mathbb{R}$ be a twice differentiable mapping such that there exist real constants $m$ and $M$ so that $m \leqslant f^{\prime \prime} \leqslant M$. Assume that $w$ : $[a, b] \rightarrow \mathbb{R}$ is nonnegative, integrable, and symmetric about $x=(a+b) / 2$. Then

$$
\begin{aligned}
& \frac{m}{2} \int_{a}^{b}(t-a)(b-a) w(t) \mathrm{d} t \\
& \quad \leqslant \frac{f(a)+f(b)}{2} \int_{a}^{b} w(t) \mathrm{d} t-\int_{a}^{b} f(t) w(t) \mathrm{d} t \\
& \quad \leqslant \frac{M}{2} \int_{a}^{b}(t-a)(b-a) w(t) \mathrm{d} t,
\end{aligned}
$$

and

$$
\begin{aligned}
& \frac{m}{8} \int_{a}^{b}(2 t-a-b)^{2} w(t) \mathrm{d} t \\
& \quad \leqslant \int_{a}^{b} f(t) w(t) \mathrm{d} t-f\left(\frac{a+b}{2}\right) \int_{a}^{b} w(t) \mathrm{d} t \\
& \quad \leqslant \frac{M}{8} \int_{a}^{b}(2 t-a-b)^{2} w(t) \mathrm{d} t .
\end{aligned}
$$

Tariboon and Ntouyas introduced quantum calculus on finite intervals in Ref. 14 . Noor et $\mathrm{al}^{15}$ applied a quantum analogue of the classical integral identity to establish some quantum estimates for Hermite-Hadamard inequalities for $q$-differentiable convex functions and $q$-differentiable quasi-convex functions. Tariboon and Ntouyas ${ }^{16}$ extended the Hölder, Hermite-Hadamard, trapezoid, Ostrowski, Cauchy-Bunyakovsky-Schwarz, Grüss, and GrüssČebyšev integral inequalities to quantum calculus on finite intervals. Sudsutad et $\mathrm{al}^{17}$ obtained some new Hermite-Hadamard type quantum integral inequalities for convex functions. Chen and Yang ${ }^{18}$ and Liu and Yang ${ }^{19}$ obtained some new Chebyshev and Grüss type inequalities via quantum integrals on finite intervals, respectively. Here we establish some new inequalities of Fejér type inequalities for differentiable mappings via quantum calculus on finite intervals. These are extensions of results given previously.

\section{PRELIMINARIES}

Definition 1 [Ref. 14] Let $I=[a, b] \subset \mathbb{R}, I^{0}=$ $(a, b)$ and $0<q<1$ be a constant. Assume $f: I \rightarrow \mathbb{R}$ is a continuous function and let $x \in I$. Then the expression

$$
\begin{aligned}
& { }_{a} D_{q} f(x)=\frac{f(x)-f_{q}(x, a)}{(1-q)(x-a)}, \quad x \neq a, \\
& { }_{a} D_{q} f(a)=\lim _{x \rightarrow a} D_{q} f(x),
\end{aligned}
$$

is called the $q$-derivative on $I$ of function $f$ at $x$.

We say that $f$ is $q$-differentiable on $I$ provided ${ }_{a} D_{q} f(x)$ exists for all $x \in I$. Note that if $a=0$ in (6), then ${ }_{0} D_{q} f=D_{q} f$, where $D_{q}$ is the well-known $q$-derivative of the function $f(x)$ defined by ${ }^{20}$

$$
D_{q} f(x)=\frac{f(x)-f(q x)}{(1-q) x} .
$$

Definition 2 [Ref. 14] Assume $f: I \rightarrow \mathbb{R}$ is a continuous function. Then the $q$-integral on $I$ is defined by

$$
\begin{aligned}
I_{q}^{a} f(x) & =\int_{a}^{x} f(t){ }_{a} \mathrm{~d}_{q} t \\
& =(1-q)(x-a) \sum_{n=0}^{\infty} q^{n} f_{q^{n}}(x, a),
\end{aligned}
$$

for $x \in I$. If $c \in(a, x)$ then the definite $q$-integral on $I$ is defined by

$$
\begin{aligned}
\int_{c}^{x} f(t)_{a} \mathrm{~d}_{q} t= & \int_{a}^{x} f(t)_{a} \mathrm{~d}_{q} t-\int_{a}^{c} f(t)_{a} \mathrm{~d}_{q} t \\
= & (1-q)(x-a) \sum_{n=0}^{\infty} q^{n} f_{q^{n}}(x, a) \\
& -(1-q)(c-a) \sum_{n=0}^{\infty} q^{n} f_{q^{n}}(c, a) .
\end{aligned}
$$

Note that if $a=0$, then (7) reduces to the classical $q$-integral of a function $f(x)$ defined by ${ }^{20}$

$$
\int_{0}^{x} f(t)_{0} \mathrm{~d}_{q} t=(1-q) x \sum_{n=0}^{\infty} q^{n} f\left(q^{n} x\right), \quad \forall x \in[0, \infty) .
$$


Lemma 1 (Ref. 18) Assume $f, g: I \rightarrow \mathbb{R}$ are two continuous functions and $f(t) \leqslant g(t)$ for all $t \in I$. Then

$$
\int_{a}^{x} f(t)_{a} \mathrm{~d}_{q} t \leqslant \int_{a}^{x} g(t)_{a} \mathrm{~d}_{q} t .
$$

Theorem 5 (Ref. 14) Let $f: I \rightarrow \mathbb{R}$ be a continuous function. Then we have

(i) ${ }_{a} D_{q} \int_{a}^{x} f(t)_{a} \mathrm{~d}_{q} t=f(x)$;

(ii) $\int_{c}^{x}{ }_{a} D_{q} f(t)_{a} \mathrm{~d}_{q} t=f(x)-f(c)$, for $c \in(a, x)$.

Theorem 6 (Ref. 14) Let $f, g: I \rightarrow \mathbb{R}$ be two continuous functions and $\alpha \in \mathbb{R}$. Then, for $x \in I$, we have

(i) $\int_{a}^{x}[f(t)+g(t)]_{a} \mathrm{~d}_{q} t$ $=\int_{a}^{x} f(t)_{a} \mathrm{~d}_{q} t+\int_{a}^{x} g(t)_{a} \mathrm{~d}_{q} t$

(ii) $\int_{a}^{x}(\alpha f)(t)_{a} \mathrm{~d}_{q} t=\alpha \int_{a}^{x} f(t)_{a} \mathrm{~d}_{q} t$;

(iii) $\int_{c}^{x} f(t)_{a} D_{q} g(t)_{a} \mathrm{~d}_{q} t=[(f g)(t)]_{c}^{x}-\int_{c}^{x} g(q t+$ $(1-q) a)_{a} D_{q} f(t){ }_{a} \mathrm{~d}_{q} t$, for $c \in(a, x)$.

\section{FEJÉR TYPE INEQUALITIES VIA QUANTUM CALCULUS ON FINITE INTERVALS}

Lemma 2 Let $f: I \rightarrow \mathbb{R}$ be a twice differentiable mapping such that there exist real constants $m$ and $M$ so that $m \leqslant f^{\prime \prime} \leqslant M$. Then, for $\lambda \in[0,1]$,

$$
\begin{aligned}
& m \frac{\lambda(1-\lambda)}{2}(b-a)^{2} \leqslant(1-\lambda) f(a)+\lambda f(b)-f_{\lambda}(b, a) \\
& \quad \leqslant M \frac{\lambda(1-\lambda)}{2}(b-a)^{2} .
\end{aligned}
$$

Proof: The proof is done in the same way as that of Theorem 3.1 in Ref. 13.

Theorem 7 Let $f: I \rightarrow \mathbb{R}$ be a twice differentiable mapping such that there exist real constants $m$ and $M$ so that $m \leqslant f^{\prime \prime} \leqslant M$. Then

$$
\begin{aligned}
& \frac{m q^{2}(b-a)^{2}}{(1+q)\left(1+q+q^{2}\right)} \\
& \quad \leqslant[f(a)+f(b)]-\frac{\int_{a}^{b}[f(x)+f(a+b-x)]_{a} \mathrm{~d}_{q} x}{b-a} \\
& \quad \leqslant \frac{M q^{2}(b-a)^{2}}{(1+q)\left(1+q+q^{2}\right)},
\end{aligned}
$$

and

$$
\begin{aligned}
& \frac{m\left(1+2 q-2 q^{2}+q^{3}\right)(b-a)^{2}}{4(1+q)\left(1+q+q^{2}\right)} \\
& \leqslant \frac{\int_{a}^{b}[f(x)+f(a+b-x)]_{a} \mathrm{~d}_{q} x}{b-a}-2 f\left(\frac{a+b}{2}\right)
\end{aligned}
$$

$$
\leqslant \frac{M\left(1+2 q-2 q^{2}+q^{3}\right)(b-a)^{2}}{4(1+q)\left(1+q+q^{2}\right)} .
$$

Proof: Integrating (8) with respect to $\lambda$ over $[0,1]$, we have

$$
\begin{aligned}
& m(b-a)^{2} \int_{0}^{1} \frac{\lambda(1-\lambda)}{2}{ }_{0} \mathrm{~d}_{q} \lambda \\
& \leqslant f(a) \int_{0}^{1}(1-\lambda)_{0} \mathrm{~d}_{q} \lambda+f(b) \int_{0}^{1} \lambda_{0} \mathrm{~d}_{q} \lambda \\
& \quad-\int_{0}^{1} f_{\lambda}(b, a){ }_{0} \mathrm{~d}_{q} \lambda \\
& \leqslant M(b-a)^{2} \int_{0}^{1} \frac{\lambda(1-\lambda)}{2}{ }_{0} \mathrm{~d}_{q} \lambda .
\end{aligned}
$$

By simple computation and changing the variable of (11), we obtain

$$
\begin{aligned}
& \frac{m q^{2}(b-a)^{2}}{2(1+q)\left(1+q+q^{2}\right)} \\
& \quad \leqslant \frac{q f(a)}{1+q}+\frac{f(b)}{1+q}-\frac{1}{b-a} \int_{a}^{b} f(x)_{a} \mathrm{~d}_{q} x \\
& \quad \leqslant \frac{M q^{2}(b-a)^{2}}{2(1+q)\left(1+q+q^{2}\right)} .
\end{aligned}
$$

Similarly, integrating the first inequality of Theorem 3 with respect to $\lambda$ over $[0,1]$, we have

$$
\begin{aligned}
& m(b-a)^{2} \int_{0}^{1} \frac{\lambda(1-\lambda)}{2}{ }_{0} \mathrm{~d}_{q} \lambda \\
& \leqslant f(a) \int_{0}^{1} \lambda_{0} \mathrm{~d}_{q} \lambda+f(b) \int_{0}^{1}(1-\lambda)_{0} \mathrm{~d}_{q} \lambda \\
& \quad-\int_{0}^{1} f_{\lambda}(a, b){ }_{0} \mathrm{~d}_{q} \lambda \\
& \leqslant M(b-a)^{2} \int_{0}^{1} \frac{\lambda(1-\lambda)}{2}{ }_{0} \mathrm{~d}_{q} \lambda .
\end{aligned}
$$

By simple computation and changing the variable of (13), we obtain

$$
\begin{aligned}
& \frac{m q^{2}(b-a)^{2}}{2(1+q)\left(1+q+q^{2}\right)} \\
& \quad \leqslant \frac{f(a)}{1+q}+\frac{q f(b)}{1+q}-\frac{1}{b-a} \int_{a}^{b} f(a+b-x)_{a} \mathrm{~d}_{q} x \\
& \quad \leqslant \frac{M q^{2}(b-a)^{2}}{2(1+q)\left(1+q+q^{2}\right)}
\end{aligned}
$$

We obtain inequality (9) from (12) and (14). Integrating the second inequality of Theorem 3 with respect to $\lambda$ over $[0,1]$ and using the change of the variable, we obtain inequalities (10). 
Remark 1 If $q^{-} \rightarrow 1$, then inequalities (9) and (10) reduce to (2) and (3), respectively.

Theorem 8 Let $f: I \rightarrow \mathbb{R}$ be a twice differentiable mapping such that there exist real constants $m$ and $M$ so that $m \leqslant f^{\prime \prime} \leqslant M$. Assume that $w: I \rightarrow \mathbb{R}$ is nonnegative, integrable, and symmetric about $x=$ $(a+b) / 2$. Then

$$
\begin{aligned}
& m \int_{a}^{b}(x-a)(b-x) w(x)_{a} \mathrm{~d}_{q} x \\
& \leqslant[f(a)+f(b)] \int_{a}^{b} w(x)_{a} \mathrm{~d}_{q} x \\
& \quad \quad-\frac{1}{b-a} \int_{a}^{b}[f(x)+f(a+b-x)] w(x)_{a} \mathrm{~d}_{q} x \\
& \leqslant M \int_{a}^{b}(x-a)(b-x) w(x)_{a} \mathrm{~d}_{q} x
\end{aligned}
$$

and

$$
\begin{aligned}
& \frac{m}{4} \int_{a}^{b}(2 x-a-b)^{2} w(x)_{a} \mathrm{~d}_{q} x \\
& \leqslant \int_{a}^{b}[f(x)+f(a+b-x)] w(x)_{a} \mathrm{~d}_{q} x \\
& \quad-2 f\left(\frac{a+b}{2}\right) \int_{a}^{b} w(x)_{a} \mathrm{~d}_{q} x \\
& \leqslant \frac{M}{4} \int_{a}^{b}(2 x-a-b)^{2} w(x)_{a} \mathrm{~d}_{q} x .
\end{aligned}
$$

Proof: Multiplying both sides of (8) by $w_{\lambda}(b, a)$, and then integrating the resulting inequality with respect to $\lambda$ over $[0,1]$, we have

$$
\begin{gathered}
m(b-a)^{2} \int_{0}^{1} \frac{\lambda(1-\lambda)}{2} w_{\lambda}(b, a)_{0} \mathrm{~d}_{q} \lambda \\
\leqslant f(a) \int_{0}^{1}(1-\lambda) w_{\lambda}(b, a)_{0} \mathrm{~d}_{q} \lambda \\
+f(b) \int_{0}^{1} \lambda w_{\lambda}(b, a)_{0} \mathrm{~d}_{q} \lambda \\
\quad-\int_{0}^{1} f_{\lambda}(b, a) w_{\lambda}(b, a)_{0} \mathrm{~d}_{q} \lambda \\
\leqslant M(b-a)^{2} \int_{0}^{1} \frac{\lambda(1-\lambda)}{2} w_{\lambda}(b, a)_{0} \mathrm{~d}_{q} \lambda .
\end{gathered}
$$

By simple computation and changing the variable of
(17), we obtain

$$
\begin{gathered}
\frac{m}{2} \int_{a}^{b}(x-a)(b-x) w(x)_{a} \mathrm{~d}_{q} x \\
\leqslant \frac{f(a)}{b-a} \int_{a}^{b}(b-x) w(x)_{a} \mathrm{~d}_{q} x \\
\quad+\frac{f(b)}{b-a} \int_{a}^{b}(x-a) w(x)_{a} \mathrm{~d}_{q} x \\
\quad-\frac{1}{b-a} \int_{a}^{b} f(x) w(x)_{a} \mathrm{~d}_{q} x \\
\leqslant \frac{M}{2} \int_{a}^{b}(x-a)(b-x) w(x)_{a} \mathrm{~d}_{q} x
\end{gathered}
$$

Similarly, multiplying both sides of the first inequality of Theorem 3 by $w_{\lambda}(b, a)$, and then integrating the resulting inequality with respect to $\lambda$ over $[0,1]$, we have

$$
\begin{aligned}
& m(b-a)^{2} \int_{0}^{1} \frac{\lambda(1-\lambda)}{2} w_{\lambda}(b, a)_{0} \mathrm{~d}_{q} \lambda \\
& \leqslant f(a) \int_{0}^{1} \lambda w_{\lambda}(b, a)_{0} \mathrm{~d}_{q} \lambda \\
& \quad+f(b) \int_{0}^{1}(1-\lambda) w_{\lambda}(b, a)_{0} \mathrm{~d}_{q} \lambda \\
& \quad-\int_{0}^{1} f_{\lambda}(a, b) w_{\lambda}(b, a)_{0} \mathrm{~d}_{q} \lambda \\
& \leqslant M(b-a)^{2} \int_{0}^{1} \frac{\lambda(1-\lambda)}{2} w_{\lambda}(a, b)_{0} \mathrm{~d}_{q} \lambda
\end{aligned}
$$

By the symmetry of $w(x)$ and changing the variable of (19), we obtain

$$
\begin{aligned}
& \frac{m}{2} \int_{a}^{b}(x-a)(b-x) w(x)_{a} \mathrm{~d}_{q} x \\
& \leqslant \frac{f(a)}{b-a} \int_{a}^{b}(x-a) w(x)_{a} \mathrm{~d}_{q} x \\
& \quad+\frac{f(b)}{b-a} \int_{a}^{b}(b-x) w(x)_{a} \mathrm{~d}_{q} x \\
& \quad-\frac{1}{b-a} \int_{a}^{b} f(a+b-x) w(x)_{a} \mathrm{~d}_{q} x \\
& \leqslant \frac{M}{2} \int_{a}^{b}(x-a)(b-x) w(x)_{a} \mathrm{~d}_{q} x .
\end{aligned}
$$

We obtain (15) from (18) and (20). Multiplying both sides of the second inequality of Theorem 3 by 
$w_{\lambda}(b, a)$, and then integrating the resulting inequality with respect to $\lambda$ over $[0,1]$, we have

$$
\begin{gathered}
m(b-a)^{2} \int_{0}^{1} \frac{(1-2 \lambda)^{2}}{8} w_{\lambda}(b, a)_{0} \mathrm{~d}_{q} \lambda \\
\leqslant \frac{1}{2}\left(\int_{0}^{1} f_{\lambda}(a, b) w_{\lambda}(b, a)_{0} \mathrm{~d}_{q} \lambda\right. \\
\left.\quad+\int_{0}^{1} f_{\lambda}(b, a) w_{\lambda}(b, a)_{0} \mathrm{~d}_{q} \lambda\right) \\
\quad-f\left(\frac{a+b}{2}\right) \int_{0}^{1} w_{\lambda}(b, a)_{0} \mathrm{~d}_{q} \lambda \\
\leqslant M(b-a)^{2} \int_{0}^{1} \frac{(1-2 \lambda)^{2}}{8} w_{\lambda}(b, a)_{0} \mathrm{~d}_{q} \lambda .
\end{gathered}
$$

By using the change of the variable of (21), we obtain

$$
\begin{aligned}
& m \int_{a}^{b} \frac{(2 x-a-b)^{2}}{8} w(x)_{a} \mathrm{~d}_{q} x \\
& \leqslant \frac{1}{2} \int_{a}^{b} f(x) w(x)_{a} \mathrm{~d}_{q} x+\frac{1}{2} \int_{a}^{b} f(a+b-x) w(x)_{a} \mathrm{~d}_{q} x \\
& \quad \quad-f\left(\frac{a+b}{2}\right) \int_{a}^{b} w(x)_{a} \mathrm{~d}_{q} x \\
& \leqslant M \int_{a}^{b} \frac{(2 x-a-b)^{2}}{8} w(x)_{a} \mathrm{~d}_{q} x,
\end{aligned}
$$

which implies (16).

Remark 2 If $q^{-} \rightarrow 1$, then inequalities (15) and (16) reduce to (4) and (5), respectively.

Lemma 3 Let $f: I \rightarrow \mathbb{R}$ be twice q-differentiable with ${ }_{a} D_{q}^{2} f$ integrable on $I$. Then

$$
\begin{aligned}
& \int_{a}^{b}(x-a)(b-x)_{a} D_{q}^{2} f(x)_{a} \mathrm{~d}_{q} x \\
& =(q b-a) f_{q}(b, a)+(b-q a) f(a) \\
& -(1+q) \int_{a}^{b} f_{q^{2}}(x, a)_{a} \mathrm{~d}_{q} x
\end{aligned}
$$

and

$$
\begin{aligned}
& \int_{a}^{b}\left[(x-a)^{2}+(x-b)^{2}\right]_{a} D_{q}^{2} f(x){ }_{a} \mathrm{~d}_{q} x \\
& \left.\quad=2(1+q) \int_{a}^{b} f_{q^{2}}(x, a)\right)_{a} \mathrm{~d}_{q} x-2(q b-a) f_{q}(b, a) \\
& \quad-2(b-q a) f(a)+(b-a)^{2}\left({ }_{a} D_{q} f(b)-{ }_{a} D_{q} f(a)\right) .
\end{aligned}
$$

Proof: By integration by parts, we have that

$$
\begin{aligned}
& \int_{a}^{b}(x-a)(b-x)_{a} D_{q}^{2} f(x)_{a} \mathrm{~d}_{q} x \\
& =\left[(x-a)(b-x)_{a} D_{q} f(x)\right]_{a}^{b} \\
& \quad-\int_{a}^{b}[(a+b)-(1+q) x]_{a} D_{q} f_{q}(x, a)_{a} \mathrm{~d}_{q} x \\
& =\quad\left[((1+q) x-(a+b)] f_{q}(x, a)\right]_{a}^{b} \\
& \quad-(1+q) \int_{a}^{b} f_{q^{2}}(x, a)_{a} \mathrm{~d}_{q} x \\
& =(q b-a) f_{q}(b, a)+(b-q a) f(a) \\
& \quad-(1+q) \int_{a}^{b} f_{q^{2}}(x, a)_{a} \mathrm{~d}_{q} x
\end{aligned}
$$

which implies (22). Next, we prove (23). Using integration by parts, we obtain

$$
\begin{gathered}
\int_{a}^{b}(x-a)^{2}{ }_{a} D_{q}^{2} f(x)_{a} \mathrm{~d}_{q} x=\left[(x-a)^{2}{ }_{a} D_{q} f(x)\right]_{a}^{b} \\
\quad-\int_{a}^{b}[(1+q) x-2 a]_{a} D_{q} f_{q}(x, a)_{a} \mathrm{~d}_{q} x \\
=(b-a)^{2}{ }_{a} D_{q} f(b)-\left[[(1+q) x-2 a] f_{q}(x, a)\right]_{a}^{b} \\
\quad+(1+q) \int_{a}^{b} f_{q^{2}}(x, a)_{a} \mathrm{~d}_{q} x \\
=(b-a)^{2}{ }_{a} D_{q} f(b)-[(1+q) b-2 a] f_{q}(b, a) \\
\quad-(1-q) a f(a)+(1+q) \int_{a}^{b} f_{q^{2}}(x, a)_{a} \mathrm{~d}_{q} x
\end{gathered}
$$

and

$$
\begin{aligned}
\int_{a}^{b}(x- & b)^{2}{ }_{a} D_{q}^{2} f(x){ }_{a} \mathrm{~d}_{q} x=\left[(x-b)^{2}{ }_{a} D_{q} f(x)\right]_{a}^{b} \\
& -\int_{a}^{b}[(1+q) x-2 b]_{a} D_{q} f_{q}(x, a)_{a} \mathrm{~d}_{q} x \\
=- & (b-a)^{2} D_{q} f(a) \\
- & {\left[[(1+q) x-2 b] f_{q}(x, a)\right]_{a}^{b} } \\
+ & (1+q) \int_{a}^{b} f_{q^{2}}(x, a)_{a} \mathrm{~d}_{q} x \\
=- & (b-a)_{a}^{2} D_{q} f(a)+(1-q) b f_{q}(b, a) \\
+ & {[(1+q) a-2 b] f(a) } \\
+ & (1+q) \int_{a}^{b} f_{q^{2}}(x, a)_{a} \mathrm{~d}_{q} x
\end{aligned}
$$


Adding (24) and (25), we deduce (23).

Theorem 9 Let $f: I \rightarrow \mathbb{R}$ be twice q-differentiable with ${ }_{a} D_{q}^{2} f$ integrable on $I$ and $m \leqslant{ }_{a} D_{q}^{2} f \leqslant M$. Then

$$
\begin{aligned}
& \frac{m q^{2}(b-a)^{3}}{(1+q)\left(1+q+q^{2}\right)} \\
& \leqslant(q b-a) f_{q}(b, a)+(b-q a) f(a) \\
& \quad-(1+q) \int_{a}^{b} f_{q^{2}}(x, a)_{a} \mathrm{~d}_{q} x \\
& \leqslant \frac{M q^{2}(b-a)^{3}}{(1+q)\left(1+q+q^{2}\right)},
\end{aligned}
$$

and

$$
\begin{aligned}
& \frac{m\left(1+q+q^{3}\right)(b-a)^{3}}{2(1+q)\left(1+q+q^{2}\right)} \\
& \left.\leqslant 2(1+q) \int_{a}^{b} f_{q^{2}}(x, a)\right)_{a} \mathrm{~d}_{q} x \\
& \quad-2(q b-a) f_{q}(b, a)-2(b-q a) f(a) \\
& \quad+(b-a)^{2}\left({ }_{a} D_{q} f(b)-{ }_{a} D_{q} f(a)\right) \\
& \leqslant \frac{M\left(1+q+q^{3}\right)(b-a)^{3}}{2(1+q)\left(1+q+q^{2}\right)} .
\end{aligned}
$$

Proof: Since $m \leqslant{ }_{a} D_{q}^{2} f \leqslant M$, we have

$$
\begin{aligned}
m(x-a)(b-x) & \leqslant(x-a)(b-x)_{a} D_{q}^{2} f(x) \\
& \leqslant M(x-a)(b-x), \quad \forall x \in[a, b] .
\end{aligned}
$$

Integrating (28) with respect to $x$ from $a$ to $b$, we obtain

$$
\begin{aligned}
& m \int_{a}^{b}(x-a)(b-x)_{a} \mathrm{~d}_{q} x \\
& \leqslant \int_{a}^{b}(x-a)(b-x)_{a} D_{q}^{2} f(x)_{a} \mathrm{~d}_{q} x \\
& \quad \leqslant M \int_{a}^{b}(x-a)(b-x)_{a} \mathrm{~d}_{q} x .
\end{aligned}
$$

However, by (22), we have

$$
\begin{aligned}
& \int_{a}^{b}(x-a)(b-x)_{a} D_{q}^{2} f(x)_{a} \mathrm{~d}_{q} x \\
& =(q b-a) f_{q}(b, a)+(b-q a) f(a) \\
& -(1+q) \int_{a}^{b} f_{q^{2}}(x, a)_{a} \mathrm{~d}_{q} x
\end{aligned}
$$

and

$$
\int_{a}^{b}(x-a)(b-x)_{a} \mathrm{~d}_{q} x=\frac{q^{2}(b-a)^{3}}{(1+q)\left(1+q+q^{2}\right)} .
$$

We obtain (26) from (29)-(31).

From $m \leqslant{ }_{a} D_{q}^{2} f \leqslant M$, we have

$$
m(x-a)^{2} \leqslant(x-a)^{2} D_{q}^{2} f(x) \leqslant M(x-a)^{2},
$$

and

$$
m(x-b)^{2} \leqslant(x-b)_{a}^{2} D_{q}^{2} f(x) \leqslant M(x-b)^{2},
$$

for $x \in[a, b]$. Integrating (32) and (33) with respect to $x$ from $a$ to $b$ we obtain

$$
\begin{aligned}
m \int_{a}^{b}(x-a)^{2}{ }_{a} \mathrm{~d}_{q} x & \leqslant \int_{a}^{b}(x-a)^{2}{ }_{a} D_{q}^{2} f(x){ }_{a} \mathrm{~d}_{q} x \\
& \leqslant M \int_{a}^{b}(x-a)^{2}{ }_{a} \mathrm{~d}_{q} x
\end{aligned}
$$

and

$$
\begin{aligned}
m \int_{a}^{b}(x-b)^{2}{ }_{a} \mathrm{~d}_{q} x & \leqslant \int_{a}^{b}(x-b)^{2}{ }_{a} D_{q}^{2} f(x){ }_{a} \mathrm{~d}_{q} x \\
& \leqslant M \int_{a}^{b}(x-b)^{2}{ }_{a} \mathrm{~d}_{q} x
\end{aligned}
$$

respectively. By simple computation, we have

$$
\begin{aligned}
& \int_{a}^{b}(x-a)^{2}{ }_{a} \mathrm{~d}_{q} x=\frac{(b-a)^{3}}{1+q+q^{2}}, \\
& \int_{a}^{b}(x-b)^{2}{ }_{a} \mathrm{~d}_{q} x=\frac{\left(q+q^{3}\right)(b-a)^{3}}{(1+q)\left(1+q+q^{2}\right)} .
\end{aligned}
$$

We obtain (27) from Lemma 3 and (34)-(36).

Theorem 10 Let $f: I \rightarrow \mathbb{R}$ be twice q-differentiable with ${ }_{a} D_{q}^{2} f$ integrable on $I$ and $m \leqslant{ }_{a} D_{q}^{2} f \leqslant M$. Then

$$
\begin{aligned}
& \mid(q b-a) f_{q}(b, a)+(b-q a) f(a) \\
& -(1+q) \int_{a}^{b} f_{q^{2}}(x, a){ }_{a} \mathrm{~d}_{q} x \\
& -\frac{q^{2}(b-a)^{2}\left({ }_{a} D_{q} f(b)-{ }_{a} D_{q} f(a)\right)}{(1+q)\left(1+q+q^{2}\right)} \mid \\
& \leqslant \frac{(b-a)^{3}}{16}(M-m) .
\end{aligned}
$$


Proof: From Theorem 3.6 in Ref. 16, we obtain

$$
\begin{gathered}
\mid \frac{1}{b-a} \int_{a}^{b}(x-a)(b-x)_{a} D_{q}^{2} f(x)_{a} \mathrm{~d}_{q} x \\
\quad-\frac{1}{(b-a)^{2}} \int_{a}^{b}(x-a)(b-x)_{a} \mathrm{~d}_{q} x \\
\times \int_{a}^{b}{ }_{a}^{2} D_{q}^{2} f(x)_{a} \mathrm{~d}_{q} x \mid \leqslant \frac{1}{4}(K-k)(M-m),
\end{gathered}
$$

where

$$
\begin{aligned}
& K=\sup _{x \in[a, b]}\{(x-a)(b-x)\}=\frac{(b-a)^{2}}{4}, \\
& k=\inf _{x \in[a, b]}\{(x-a)(b-x)\}=0 .
\end{aligned}
$$

By Lemma 3, (31), (38) and (39), we have

$$
\begin{gathered}
\mid \frac{1}{b-a}\left((q b-a) f_{q}(b, a)+(b-q a) f(a)\right. \\
\left.-(1+q) \int_{a}^{b} f_{q^{2}}(x, a){ }_{a} \mathrm{~d}_{q} x\right) \\
-\frac{q^{2}(b-a)\left({ }_{a} D_{q} f(b)-{ }_{a} D_{q} f(a)\right)}{(1+q)\left(1+q+q^{2}\right)} \mid \leqslant \frac{(b-a)^{2}}{16}(M-m),
\end{gathered}
$$

which implies (37).

Remark 3 If $q^{-} \rightarrow 1$, then (37) reduces to the result obtained in Ref. 12 .

Lemma 4 Let $\varphi, g: I \rightarrow \mathbb{R}$ be two continuous functions and $q$-differentiable on $I^{0}$. If ${ }_{a} D_{q} g(x) \neq 0$ on $I^{0}$ and $m \leqslant{ }_{a} D_{q} \varphi(x) /{ }_{a} D_{q} g(x) \leqslant M$ on $I^{0}$, then

$$
\begin{aligned}
& m\left((b-a) \int_{a}^{b} g^{2}(x)_{a} \mathrm{~d}_{q} x-\left(\int_{a}^{b} g(x)_{a} \mathrm{~d}_{q} x\right)^{2}\right) \\
& \leqslant(b-a) \int_{a}^{b} \varphi(x) g(x)_{a} \mathrm{~d}_{q} x \\
& \quad-\int_{a}^{b} \varphi(x)_{a} \mathrm{~d}_{q} x \int_{a}^{b} g(x)_{a} \mathrm{~d}_{q} x \\
& \leqslant M\left((b-a) \int_{a}^{b} g^{2}(x)_{a} \mathrm{~d}_{q} x-\left(\int_{a}^{b} g(x)_{a} \mathrm{~d}_{q} x\right)^{2}\right) .
\end{aligned}
$$

Proof: If ${ }_{a} D_{q} g(x) \geqslant 0$, then $g(x)$ is an increasing function and

$$
m_{a} D_{q} g(x) \leqslant{ }_{a} D_{q} \varphi(x) \leqslant M_{a} D_{q} g(x),
$$

for $\forall x \in I^{0}$. For $a \leqslant x \leqslant y \leqslant b$, integrating (41) with respect to $x$ from $x$ to $y$, we obtain

$$
m(g(y)-g(x)) \leqslant \varphi(y)-\varphi(x) \leqslant M(g(y)-g(x)) .
$$

Multiplying both sides of (42) by $g(y)-g(x) \geqslant 0$, then

$$
\begin{aligned}
m(g(y)-g(x))^{2} & \leqslant(\varphi(y)-\varphi(x))(g(y)-g(x)) \\
& \leqslant M(g(y)-g(x))^{2} .
\end{aligned}
$$

Similarly, if ${ }_{a} D_{q} g(x) \leqslant 0$, we can also obtain (43). Integrating the obtained result (43) with respect to $x$ and $y$ from $a$ to $b$, we have

$$
\begin{aligned}
& m \int_{a}^{b} \int_{a}^{b}(g(y)-g(x))^{2}{ }_{a} \mathrm{~d}_{q} x_{a} \mathrm{~d}_{q} y \\
& \quad \leqslant \int_{a}^{b} \int_{a}^{b}(\varphi(y)-\varphi(x))(g(y)-g(x))_{a} \mathrm{~d}_{q} x_{a} \mathrm{~d}_{q} y \\
& \quad \leqslant M \int_{a}^{b} \int_{a}^{b}(g(y)-g(x))^{2}{ }_{a} \mathrm{~d}_{q} x_{a} \mathrm{~d}_{q} y .
\end{aligned}
$$

A simple calculation shows us that

$$
\begin{aligned}
\int_{a}^{b} \int_{a}^{b}(g(y)-g(x))^{2}{ }_{a} \mathrm{~d}_{q} x_{a} \mathrm{~d}_{q} y \\
=2\left[(b-a) \int_{a}^{b} g^{2}(x)_{a} \mathrm{~d}_{q} x\right. \\
\left.-\left(\int_{a}^{b} g(x)_{a} \mathrm{~d}_{q} x\right)^{2}\right],
\end{aligned}
$$

and

$$
\begin{aligned}
\int_{a}^{b} \int_{a}^{b}(\varphi(y)-\varphi(x))(g(y)-g(x))_{a} \mathrm{~d}_{q} x \mathrm{~d}_{q} y \\
=2\left((b-a) \int_{a}^{b} \varphi(x) g(x)_{a} \mathrm{~d}_{q} x\right. \\
\left.\quad-\int_{a}^{b} \varphi(x)_{a} \mathrm{~d}_{q} x \int_{a}^{b} g(x)_{a} \mathrm{~d}_{q} x\right) .
\end{aligned}
$$

We obtain (40) from (44)-(46).

Lemma 5 Let $f: I \rightarrow \mathbb{R}$ be twice q-differentiable with ${ }_{a} D_{q} f$ integrable on $I$. Then

$$
\begin{aligned}
& \left.\int_{a}^{b}(2 x-a-b)_{a} D_{q} f(x)\right)_{a} \mathrm{~d}_{q} x \\
= & (b-a)(f(a)+f(b))-2 \int_{a}^{b} f_{q}(x, a)_{a} \mathrm{~d}_{q} x .
\end{aligned}
$$


Proof: The proof is similar to that of (23) in Lemma 3.

Theorem 11 Let $f: I \rightarrow \mathbb{R}$ be twice q-differentiable with ${ }_{a} D_{q}^{2} f$ integrable on $I$ and $m \leqslant{ }_{a} D_{q}^{2} f \leqslant M$. Then

$$
\begin{aligned}
& \frac{m q(b-a)^{3}}{(1+q)^{2}\left(1+q+q^{2}\right)} \\
& \leqslant \frac{(b-a)((1+q) f(a)+2 q f(b))}{2(1+q)} \\
& \quad-\int_{a}^{b} f_{q}(x, a)_{a} \mathrm{~d}_{q} x \\
& \leqslant \frac{M q(b-a)^{3}}{(1+q)^{2}\left(1+q+q^{2}\right)} .
\end{aligned}
$$

Proof: Method 1. From Lemma 4, we choose $\varphi(x)={ }_{a} D_{q} f(x)$ and $g(x)=x-\frac{1}{2}(a+b)$. Then $m \leqslant{ }_{a} D_{q} \varphi(x) /{ }_{a} D_{q} g(x) \leqslant M$ holds on $(a, b)$. By Lemma 4,

$$
\begin{gathered}
m\left((b-a) \int_{a}^{b}\left(x-\frac{a+b}{2}\right)^{2}{ }_{a} \mathrm{~d}_{q} x\right. \\
\left.\quad-\left(\int_{a}^{b}\left(x-\frac{a+b}{2}\right)_{a} \mathrm{~d}_{q} x\right)^{2}\right) \\
\leqslant(b-a) \int_{a}^{b}\left(x-\frac{a+b}{2}\right)_{a} D_{q} f(x)_{a} \mathrm{~d}_{q} x \\
\quad-\int_{a}^{b} D_{q} f(x)_{a} \mathrm{~d}_{q} x \int_{a}^{b}\left(x-\frac{a+b}{2}\right)_{a} \mathrm{~d}_{q} x \\
\leqslant M\left((b-a) \int_{a}^{b}\left(x-\frac{a+b}{2}\right)^{2}{ }_{a} \mathrm{~d}_{q} x\right. \\
\left.-\left(\int_{a}^{b}\left(x-\frac{a+b}{2}\right)_{a} \mathrm{~d}_{q} x\right)^{2}\right) .
\end{gathered}
$$

A simple calculation shows that

$$
\begin{aligned}
\int_{a}^{b} y(x)_{a} \mathrm{~d}_{q} x & =\frac{(1-q)(b-a)^{2}}{2(1+q)} \\
\int_{a}^{b} y(x)^{2}{ }_{a} \mathrm{~d}_{q} x & =\frac{\left(1+2 q-2 q^{2}+q^{3}\right)(b-a)^{3}}{4(1+q)\left(1+q+q^{2}\right)},
\end{aligned}
$$

where $y(x)=x+(a+b) / 2$, and

$$
\begin{aligned}
& \int_{a}^{b}\left(x-\frac{a+b}{2}\right){ }_{a} D_{q} f(x)_{a} \mathrm{~d}_{q} x \\
& =\frac{(b-a)(f(a)+f(b))}{2}-\int_{a}^{b} f_{q}(x, a)_{a} \mathrm{~d}_{q} x .
\end{aligned}
$$

We obtain (48) from (49)-(51).
Method 2. The identity (47) is expressed as

$$
\begin{gathered}
(b-a)(f(a)+f(b))-2 \int_{a}^{b} f_{q}(x, a)_{a} \mathrm{~d}_{q} x \\
=\int_{a}^{b}(x-a)_{a} D_{q} f(x)_{a} \mathrm{~d}_{q} x \\
+\int_{a}^{b}(x-b)_{a} D_{q} f(x)_{a} \mathrm{~d}_{q} x .
\end{gathered}
$$

Now we apply Lemma 4 to obtain

$$
\begin{aligned}
& m\left((b-a) \int_{a}^{b}(x-a)^{2}{ }_{a} \mathrm{~d}_{q} x-\Lambda_{a}^{2}\right) \\
& \leqslant(b-a) \int_{a}^{b}(x-a)_{a} D_{q} f(x)_{a} \mathrm{~d}_{q} x \\
& \quad-\Lambda_{a} \int_{a}^{b} D_{q} f(x)_{a} \mathrm{~d}_{q} x \\
& \leqslant M\left((b-a) \int_{a}^{b}(x-a)^{2}{ }_{a} \mathrm{~d}_{q} x-\Lambda_{a}^{2}\right) .
\end{aligned}
$$

where $\Lambda_{a}=\int_{a}^{b}(x-a)_{a} \mathrm{~d}_{q} x$. A simple calculation and (ii) of Theorem 3.2 in Ref. 14 show that

$$
\left.\begin{array}{rl}
\int_{a}^{b}(x-a)_{a} \mathrm{~d}_{q} x & =\frac{(b-a)^{2}}{1+q}, \\
\int_{a}^{b}(x-a)^{2}{ }_{a} \mathrm{~d}_{q} x & =\frac{(b-a)^{3}}{1+q+q^{2}}, \\
\int_{a}^{b}{ }_{a} D_{q} f(x)_{a} \mathrm{~d}_{q} x & =f(b) .
\end{array}\right\}
$$

From (53) and (54), we obtain

$$
\begin{aligned}
& \frac{m q(b-a)^{4}}{(1+q)^{2}\left(1+q+q^{2}\right)} \\
& \leqslant(b-a) \int_{a}^{b}(x-a)_{a} D_{q} f(x)_{a} \mathrm{~d}_{q} x \\
& \quad-\frac{(b-a)^{2}}{1+q} f(b) \\
& \leqslant \frac{M q(b-a)^{4}}{(1+q)^{2}\left(1+q+q^{2}\right)}
\end{aligned}
$$

which implies

$$
\begin{aligned}
& \frac{m q(b-a)^{3}}{(1+q)^{2}\left(1+q+q^{2}\right)} \\
& \quad \leqslant \int_{a}^{b}(x-a)_{a} D_{q} f(x)_{a} \mathrm{~d}_{q} x-\frac{b-a}{1+q} f(b) \\
& \quad \leqslant \frac{M q(b-a)^{3}}{(1+q)^{2}\left(1+q+q^{2}\right)} .
\end{aligned}
$$


Similarly, Lemma 4 gives

$$
\begin{aligned}
& m\left((b-a) \int_{a}^{b}(x-b)^{2}{ }_{a} \mathrm{~d}_{q} x-\Lambda_{b}^{2}\right) \\
& \leqslant(b-a) \int_{a}^{b}(x-b)_{a} D_{q} f(x)_{a} \mathrm{~d}_{q} x \\
& \quad-\Lambda_{b} \int_{a}^{b} D_{a} D_{q} f(x)_{a} \mathrm{~d}_{q} x \\
& \leqslant M\left((b-a) \int_{a}^{b}(x-b)^{2}{ }_{a} \mathrm{~d}_{q} x-\Lambda_{b}^{2}\right),
\end{aligned}
$$

where $\Lambda_{b}=\int_{a}^{b}(x-b){ }_{a} \mathrm{~d}_{q} x$. A simple calculation shows that

$$
\begin{aligned}
& \int_{a}^{b}(x-b)_{a} \mathrm{~d}_{q} x=-\frac{q(b-a)^{2}}{1+q}, \\
& \int_{a}^{b}(x-b)^{2}{ }_{a} \mathrm{~d}_{q} x=\frac{\left(q+q^{3}\right)(b-a)^{3}}{(1+q)\left(1+q+q^{2}\right)} .
\end{aligned}
$$

From (56) and (57), we obtain

$$
\begin{aligned}
& \frac{m q(b-a)^{4}}{(1+q)^{2}\left(1+q+q^{2}\right)} \\
& \leqslant(b-a) \int_{a}^{b}(x-b)_{a} D_{q} f(x)_{a} \mathrm{~d}_{q} x \\
& \quad+\frac{q(b-a)^{2}}{1+q} f(b) \\
& \leqslant \frac{M q(b-a)^{4}}{(1+q)^{2}\left(1+q+q^{2}\right)}
\end{aligned}
$$

which implies

$$
\begin{aligned}
& \frac{m q(b-a)^{3}}{(1+q)^{2}\left(1+q+q^{2}\right)} \\
& \quad \leqslant \int_{a}^{b}(x-b)_{a} D_{q} f(x)_{a} \mathrm{~d}_{q} x+\frac{q(b-a)}{1+q} f(b) \\
& \quad \leqslant \frac{M q(b-a)^{3}}{(1+q)^{2}\left(1+q+q^{2}\right)} .
\end{aligned}
$$

We now add (55) and (58), taking into account the identity (52). Then we obtain (48) from Lemma 5.

Remark 4 From Theorem 11, we obtain the same result from two different methods.

Theorem 12 Let $f: I \rightarrow \mathbb{R}$ be twice q-differentiable with ${ }_{a} D_{q}^{2} f$ integrable on $I$ and $m \leqslant{ }_{a} D_{q}^{2} f \leqslant M$. Then

$$
\begin{aligned}
& \frac{M q^{2}(b-a)^{3}}{(1+q)\left(1+q+q^{2}\right)}+B \\
& \leqslant(q b-a) f_{q}(b, a)+(b-q a) f(a) \\
& \quad-(1+q) \int_{a}^{b} f_{q^{2}}(x, a){ }_{a} \mathrm{~d}_{q} x \\
& \leqslant A+\frac{m q^{2}(b-a)^{3}}{(1+q)\left(1+q+q^{2}\right)}
\end{aligned}
$$

where

$$
\begin{aligned}
A=\left[(b-a)\left({ }_{a} D_{q} f(b)-\frac{m(b-a)}{1+q}\right)\right. & \left.-\left(f_{q}(b, a)-f(a)\right)\right] \\
& \times\left\{\frac{f_{q}(b, a)-f(a)}{\left({ }_{a} D_{q} f(b)-{ }_{a} D_{q} f(a)\right)-m(b-a)}\right. \\
& \left.-\frac{(b-a)\left({ }_{a} D_{q} f(a)-\frac{m q(b-a)}{1+q}\right)}{\left({ }_{a} D_{q} f(b)-{ }_{a} D_{q} f(a)\right)-m(b-a)}\right\},
\end{aligned}
$$

and

$$
\begin{gathered}
B=\left[(b-a)\left(\frac{M(b-a)}{1+q}-{ }_{a} D_{q} f(b)\right)\right. \\
\left.+\left(f_{q}(b, a)-f(a)\right)\right] \\
\times\left(\frac{f_{q}(b, a)-f(a)}{M(b-a)-\left({ }_{a} D_{q} f(b)-{ }_{a} D_{q} f(a)\right)}\right. \\
\left.\quad-\frac{(b-a)\left(\frac{M q(b-a)}{1+q}+{ }_{a} D_{q} f(a)\right)}{M(b-a)-\left({ }_{a} D_{q} f(b)-{ }_{a} D_{q} f(a)\right)}\right),
\end{gathered}
$$

provided that ${ }_{a} D_{q} f(b)-{ }_{a} D_{q} f(a) \neq m(b-a)$ and ${ }_{a} D_{q} f(b)-{ }_{a} D_{q} f(a) \neq M(b-a)$.

Proof: To prove (59), we firstly give the following weighted Chebyshev type quantum integral inequality on finite intervals:

$$
\begin{aligned}
& \int_{a}^{b} \phi(x)_{a} \mathrm{~d}_{q} x \int_{a}^{b} \phi(x) g(x) h(x){ }_{a} \mathrm{~d}_{q} x \\
& \quad \leqslant \int_{a}^{b} \phi(x) g(x)_{a} \mathrm{~d}_{q} x \int_{a}^{b} \phi(x) h(x)_{a} \mathrm{~d}_{q} x,
\end{aligned}
$$

where $g$ and $h$ are continuous asynchronous functions and $\phi$ is a continuous nonnegative function. Two functions $g$ and $h$ are said to be asynchronous on $[a, b]$, if $(g(x)-g(y))(h(x)-h(y)) \leqslant 0$, for 
any $x, y \in[a, b]$. In fact, from Definition 2 and following the proof of Lemma 3.1 in Ref. 16, we have

$$
\begin{gathered}
\int_{a}^{b} \int_{a}^{b} \phi(x) \phi(y)(g(y)-g(x))(h(y)-h(x))_{a} \mathrm{~d}_{q} x{ }_{a} \mathrm{~d}_{q} y \\
\quad=2\left(\int_{a}^{b} \phi(x)_{a} \mathrm{~d}_{q} x \int_{a}^{b} \phi(x) g(x) h(x)_{a} \mathrm{~d}_{q} x\right. \\
\left.\quad-\int_{a}^{b} \phi(x) g(x)_{a} \mathrm{~d}_{q} x \int_{a}^{b} \phi(x) h(x)_{a} \mathrm{~d}_{q} x\right) .
\end{gathered}
$$

Since $g$ and $h$ are continuous asynchronous functions and $\phi$ is continuous nonnegative function, we have

$$
\phi(x) \phi(y)(g(y)-g(x))(h(y)-h(x)) \leqslant 0 .
$$

From (61) and (62), we obtain (60).

Now let

$$
\begin{gathered}
\Omega=\int_{a}^{b}(x-a)(b-x)_{a} D_{q}^{2} f(x)_{a} \mathrm{~d}_{q} x \\
=(q b-a) f_{q}(b, a)+(b-q a) f(a) \\
\quad-(1+q) \int_{a}^{b} f_{q^{2}}(x, a)_{a} \mathrm{~d}_{q} x .
\end{gathered}
$$

From Lemma 3 we can easily obtain

$$
\begin{aligned}
& \int_{a}^{b}(x-a)(b-x)\left({ }_{a} D_{q}^{2} f(x)-m\right)_{a} \mathrm{~d}_{q} x \\
& =\Omega-m \int_{a}^{b}(x-a)(b-x){ }_{a} \mathrm{~d}_{q} x \\
& =\Omega-\frac{m q^{2}(b-a)^{3}}{(1+q)\left(1+q+q^{2}\right)}
\end{aligned}
$$

and

$$
\begin{aligned}
& \int_{a}^{b}(x-a)(b-x)\left(M-{ }_{a} D_{q}^{2} f(x)\right)_{a} \mathrm{~d}_{q} x \\
& \quad=M \int_{a}^{b}(x-a)(b-x){ }_{a} \mathrm{~d}_{q} x-\Omega \\
& \quad=\frac{M q^{2}(b-a)^{3}}{(1+q)\left(1+q+q^{2}\right)}-\Omega .
\end{aligned}
$$

From (60), we obtain

$$
\begin{aligned}
\int_{a}^{b}(x-a)(b-x)\left({ }_{a} D_{q}^{2} f(x)-m\right){ }_{a} \mathrm{~d}_{q} x \\
\leqslant \int_{a}^{b}(x-a)\left({ }_{a} D_{q}^{2} f(x)-m\right){ }_{a} \mathrm{~d}_{q} x \\
\times \frac{\int_{a}^{b}(b-x)\left({ }_{a} D_{q}^{2} f(x)-m\right){ }_{a} \mathrm{~d}_{q} x}{\int_{a}^{b}\left({ }_{a} D_{q}^{2} f(x)-m\right){ }_{a} \mathrm{~d}_{q} x},
\end{aligned}
$$

and

$$
\begin{aligned}
& \int_{a}^{b}(x-a)(b-x)\left(M-{ }_{a} D_{q}^{2} f(x)\right){ }_{a} \mathrm{~d}_{q} x \\
& \leqslant \int_{a}^{b}(x-a)\left(M-{ }_{a} D_{q}^{2} f(x)\right)_{a} \mathrm{~d}_{q} x \\
& \times \frac{\int_{a}^{b}(b-x)\left(M-{ }_{a} D_{q}^{2} f(x)\right){ }_{a} \mathrm{~d}_{q} x}{\int_{a}^{b}\left(M-{ }_{a} D_{q}^{2} f(x)\right){ }_{a} \mathrm{~d}_{q} x} .
\end{aligned}
$$

A simple calculation shows us that

$$
\begin{aligned}
& \int_{a}^{b} \int_{a}^{b}\left({ }_{a} D_{q}^{2} f(x)-m\right){ }_{a} \mathrm{~d}_{q} x \\
& \quad=\int_{a}^{b} D_{q}\left({ }_{a} D_{q} f(x)-m x\right){ }_{a} \mathrm{~d}_{q} x \\
& \quad=\left({ }_{a} D_{q} f(b)-{ }_{a} D_{q} f(a)\right)-m(b-a),
\end{aligned}
$$

$$
\begin{aligned}
& \int_{a}^{b}(x-a)\left({ }_{a} D_{q}^{2} f(x)-m\right){ }_{a} \mathrm{~d}_{q} x \\
& =\int_{a}^{b}(x-a){ }_{a} D_{q}\left({ }_{a} D_{q} f(x)-m x\right){ }_{a} \mathrm{~d}_{q} x \\
& =\left.(x-a)\left({ }_{a} D_{q} f(x)-m x\right)\right|_{a} ^{b} \\
& \quad \quad-\int_{a}^{b}\left({ }_{a} D_{q} f_{q}(x+(1-q) a)-m(q x, a)\right){ }_{a} \mathrm{~d}_{q} x \\
& =(b-a)\left({ }_{a} D_{q} f(b)-m b\right) \\
& \quad-\left(f_{q}(b, a)-f(a)-\frac{m(b-a)(b q+a)}{1+q}\right) \\
& =(b-a)\left({ }_{a} D_{q} f(b)\right. \\
& \left.\quad-\frac{m(b-a)}{1+q}\right)-\left(f_{q}(b, a)-f(a)\right),
\end{aligned}
$$


and

$$
\begin{aligned}
& \int_{a}^{b}(b-x)\left({ }_{a} D_{q}^{2} f(x)-m\right){ }_{a} \mathrm{~d}_{q} x \\
& =\int_{a}^{b}(b-x){ }_{a} D_{q}\left({ }_{a} D_{q} f(x)-m x\right){ }_{a} \mathrm{~d}_{q} x \\
& =\left.(b-x)\left({ }_{a} D_{q} f(x)-m x\right)\right|_{a} ^{b} \\
& \quad+\int_{a}^{b}\left({ }_{a} D_{q} f_{q}(x+(1-q) a)-m(q x, a)\right){ }_{a} \mathrm{~d}_{q} x \\
& =-(b-a)\left({ }_{a} D_{q} f(a)-m a\right) \\
& \quad+\left(f_{q}(b, a)-f(a)-\frac{m(b-a)(b q+a)}{1+q}\right) \\
& =\left(f_{q}(b, a)-f(a)\right) \\
& \quad-(b-a)\left({ }_{a} D_{q} f(a)-\frac{m q(b-a)}{1+q}\right) .
\end{aligned}
$$

From (63), (65), (67)-(69), we have

$$
\begin{array}{r}
\Omega-\frac{m q^{2}(b-a)^{3}}{(1+q)\left(1+q+q^{2}\right)} \leqslant\left[( b - a ) \left({ }_{a} D_{q} f(b)\right.\right. \\
\left.\left.-\frac{m(b-a)}{1+q}\right)-\left(f_{q}(b, a)-f(a)\right)\right] \\
\quad \times\left(\frac{f_{q}(b, a)-f(a)}{\left({ }_{a} D_{q} f(b)-{ }_{a} D_{q} f(a)\right)-m(b-a)}\right. \\
\left.-\frac{(b-a)\left({ }_{a} D_{q} f(a)-\frac{m q(b-a)}{1+q}\right)}{\left({ }_{a} D_{q} f(b)-{ }_{a} D_{q} f(a)\right)-m(b-a)}\right) .
\end{array}
$$

Similarly, we have

$$
\begin{aligned}
& \int_{a}^{b} \int_{a}^{b}\left(M-{ }_{a} D_{q}^{2} f(x)\right){ }_{a} \mathrm{~d}_{q} x \\
& =\int_{a}^{b}{ }_{a} D_{q}\left(M x-{ }_{a} D_{q} f(x)\right){ }_{a} \mathrm{~d}_{q} x \\
& =M(b-a)-\left({ }_{a} D_{q} f(b)-{ }_{a} D_{q} f(a)\right), \\
\int_{a}^{b}(x-a)\left(M-{ }_{a} D_{q}^{2} f(x)\right){ }_{a} \mathrm{~d}_{q} x & \\
= & \int_{a}^{b}(x-a){ }_{a} D_{q}\left(M x-{ }_{a} D_{q} f(x)\right){ }_{a} \mathrm{~d}_{q} x \\
= & \left.(x-a)\left(M x-{ }_{a} D_{q} f(x)\right)\right|_{a} ^{b} \\
& -\int_{a}^{b}\left(M(q x+(1-q) a)-{ }_{a} D_{q} f_{q}(x, a)\right){ }_{a} \mathrm{~d}_{q} x \\
= & (b-a)\left(M b-{ }_{a} D_{q} f(b)\right)-\left(\frac{M(b-a)(b q+a)}{1+q}\right. \\
& \left.\quad-\left(f_{q}(b, a)-f(a)\right)\right)
\end{aligned}
$$

We now obtain (59) from (70) and (74).

Remark 5 If $q^{-} \rightarrow 1$, then Lemma 4-Theorem 12 reduce to the results obtained in Ref. 2 .

Remark 6 Let $f(x)=x^{2} \in[a, b]$. It is easy to see that $f: I \rightarrow \mathbb{R}$ is a twice differentiable mapping such that $f^{\prime \prime}=2$. From Theorem 7, we have

$$
\begin{aligned}
& \frac{2 q^{2}(b-a)^{2}}{(1+q)\left(1+q+q^{2}\right)} \\
& \quad \leqslant\left(a^{2}+b^{2}\right)-\frac{\left(a^{2}+b^{2}\right)\left(1+2 q+q^{3}\right)+4 q^{2} a b}{(1+q)\left(1+q+q^{2}\right)} \\
& \quad \leqslant \frac{2 q^{2}(b-a)^{2}}{(1+q)\left(1+q+q^{2}\right)}
\end{aligned}
$$


and

$$
\begin{aligned}
& \frac{\left(1+2 q-2 q^{2}+q^{3}\right)(b-a)^{2}}{2(1+q)\left(1+q+q^{2}\right)} \\
& \quad \leqslant \frac{\left(a^{2}+b^{2}\right)\left(1+2 q+q^{3}\right)+4 q^{2} a b}{(1+q)\left(1+q+q^{2}\right)}-2\left(\frac{a+b}{2}\right)^{2} \\
& \leqslant \frac{\left(1+2 q-2 q^{2}+q^{3}\right)(b-a)^{2}}{2(1+q)\left(1+q+q^{2}\right)} .
\end{aligned}
$$

It is easy to see that inequalities (75) and (76) reduce to two equalities.

Remark 7 Let $f(x)=x^{3} \in[a, b]$. Then $(3-q+$ $\left.4 q^{2}\right) a=m \leqslant{ }_{a} D_{q}^{2} f \leqslant M=(1-q)\left(2-q+q^{2}\right) a+(1+$ $q)\left(1+q+q^{2}\right) b$. From Theorem 11, we have

$$
\begin{gathered}
\frac{m q(b-a)^{2}}{(1+q)^{2}\left(1+q+q^{2}\right)} \leqslant \frac{(1+q) a^{3}+2 q b^{3}}{2(1+q)} \\
-\left(\frac{q^{3}(b-a)^{3}}{1+q+q^{2}+q^{3}}+\frac{3 q^{2} a(b-a)^{2}}{1+q+q^{2}}\right. \\
\left.+\frac{3 q a^{2}(b-a)}{1+q}+a^{3}\right) \leqslant \frac{M q(b-a)^{2}}{(1+q)^{2}\left(1+q+q^{2}\right)} .
\end{gathered}
$$

\section{REFERENCES}

1. Pečarić J, Proschan F, Tong YL (1991) Convex Functions, Partial Ordering and Statistical Applications, Academic Press, New York.

2. Dragomir SS, Pearce CEM (2000) Selected Topics on Hermite-Hadamard Inequalities and Applications, RGMIA Monographs, Victoria Univ.

3. Dragomir SS (2011) Hermite-Hadamard's type inequalities for operator convex functions. Appl Math Comput 218, 766-72.

4. Sarikaya MZ, Kiris ME (2015) New inequalities of Hermite-Hadamard type for $s$-convex functions. Miskolc Math Notes 16, 491-501.

5. Xi B, Qi F, Zhang Y (2015) Some inequalities of Hermite-Hadamard type for $m$-harmonic-arithmetically convex functions. Sci Asia 41, 357-61.

6. Noor MA, Noor KI, Awan MU (2014) HermiteHadamard inequalities for relative semi-convex functions and applications. Filomat 28, 221-30.

7. Xi B, Qi F (2015) Inequalities of Hermite-Hadamard type for extended $s$-convex functions and applications to means. J Nonlin Convex Anal 16, 873-90.

8. Chun L, Qi F (2013) Integral inequalities of HermiteHadamard type for functions whose third derivatives are convex. J Inequal Appl 2013, 451.

9. Yang W (2016) Hermite-Hadamard type inequalities for $\left(p_{1}, h_{1}\right)-\left(p_{2}, h_{2}\right)$-convex functions on the coordinates. Tamkang J Math 47, 289-322.

10. Fejér L (1906) Über die Fourierreihen, II. Math. Naturwiss. Anz Ungar Akad Wiss 24, 369-90 [in Hungarian].
11. Dragomir SS, Cerone P, Sofo A (2000) Some remarks on the midpoint rule in numerical integration. Studia Univ Babes Bolyai Math XLV, 63-74.

12. Dragomir SS, Cerone P, Sofo A (2000) Some remarks on the trapezoid rule in numerical integration. Indian J Pure Appl Math 31, 475-94.

13. Minculete N, Mitroi FC (2012) Fejér-type inequalities. Aust J Math Anal Appl 9, Art. 12.

14. Tariboon J, Ntouyas SK (2013) Quantum calculus on finite intervals and applications to impulsive difference equations. Adv Difference Equat 2013, 282.

15. Noor MA, Noor KI, Awan MU (2015) Some quantum estimates for Hermite-Hadamard inequalities. Appl Math Comput 251, 675-9.

16. Tariboon J, Ntouyas SK (2014) Quantum integral inequalities on finite intervals. $J$ Inequal Appl 2014, 121.

17. Sudsutad W, Ntouyas SK, Tariboon J (2015) Quantum integral inequalities for convex functions. $J$ Math Inequal 9, 781-93.

18. Chen F, Yang W (2016) Some new Chebyshev type quantum integral inequalities on finite intervals. J Comput Anal Appl 21, 417-26.

19. Liu Z, Yang W (2016) Some new Grüss type quantum integral inequalities on finite intervals. J Nonlin Sci Appl 9, 3362-75.

20. Kac V, Cheung P (2002) Quantum Calculus, Springer, New York. 